Relations industrielles

Industrial Relations

\title{
Murray, Gregor et Pierre Verge, La représentation syndicale: visage juridique actuel et futur
}

\section{Dimitri Weiss}

Volume 55, numéro 1, 2000

URI : https://id.erudit.org/iderudit/051296ar

DOI : https://doi.org/10.7202/051296ar

Aller au sommaire du numéro

Éditeur(s)

Département des relations industrielles de l'Université Laval

ISSN

0034-379X (imprimé)

1703-8138 (numérique)

Découvrir la revue

Citer ce compte rendu

Weiss, D. (2000). Compte rendu de [Murray, Gregor et Pierre Verge, $L a$

représentation syndicale: visage juridique actuel et futur]. Relations industrielles

/ Industrial Relations, 55(1), 171-173. https://doi.org/10.7202/051296ar

Tous droits réservés @ C Département des relations industrielles de l'Universite Laval, 2000
Ce document est protégé par la loi sur le droit d'auteur. L’utilisation des services d'Érudit (y compris la reproduction) est assujettie à sa politique d'utilisation que vous pouvez consulter en ligne.

https://apropos.erudit.org/fr/usagers/politique-dutilisation/ 


\title{
Recensions
}

\section{Book Reviews}

\author{
La représentation syndicale : visage juridique actuel et futur \\ par Gregor MURRAY et Pierre VERGE, Sainte-Foy: Les Presses de \\ l'Université Laval, 1999, 182 + XII p., ISBN 2-7637-7624-8.
}

La production universitaire québécoise dans le domaine syndical est riche d'apports de qualité, notamment sous l'angle juridique, et ce dans un contexte qui a été moins défavorable à l'expression collective des salariés que dans d'autres pays, proches ou lointains. Fallait-il ajouter à ce déploiement d'écrits, dont certains marquants, un nouvel ouvrage, qui reprend une thématique fréquemment abordée ? La réponse est évidente, dans les conditions d'une mondialisation de plus en plus affirmée, d'une moindre protection des personnes au sein d'une compétition économique exacerbée et d'un accroissement du pouvoir des firmes multi (trans, inter) nationales, y compris face aux gouvernements nationaux. Elle l'est encore plus à la lecture d'un livre qui s'ingénie non seulement à dresser un inventaire pertinent, mais à analyser le devenir d'une représentation qui demeure, de par sa fonction d'intermédiation et malgré ses imperfections et ses erreurs, une composante de la démocratie.

Nous voici au tournant du siècle, qui confirme, entre autres, le décalage entre le droit et des pratiques qui participent du modèle de la flexibilité de marché ou de celui de la profession(nalité), lorsqu'il ne s'agit encore de celui de la stabilité polyvalente où les syndicats occupent traditionnellement toute leur place. L'éclatement des relations du travail est, comme ont dit, un « donné », et, du même coup, un vrai défi pour des organisations collectives, qui n'ont pas trouvé partout, aussi vite ou assez tôt, les voies du renouvellement et de l'adaptation.

Ce nouvel ouvrage commun est dû à Gregor Murray, dont j'avais écrit, ici même (Relat. ind., 1997, vol. 52, n 3, p. 654-655) l'estime que je portais au volume d'actes qu'il avait codirigé sur «L'état des relations professionnelles » (PUL, 1997), et à Pierre Verge, qui y a participé, coauteur, dernièrement, de l'excellent «Un droit du travail ? Essai sur la spécificité du droit du travail » (Éditions Yvon Blais, 1997), qui venait après une déjà longue série de contributions qui illustraient, à la fois, sa science du droit et l'apport que, en tant que juriste du travail, il apportait aux relations industrielles. Les deux auteurs qui, à partir de moments différents, ont construit chacun une œuvre, consolidée pour l'un, plus récente pour l'autre, s'étaient déjà associés, à plusieurs reprises, dans un livre (Verge et Murray, « Le droit et les syndicats $\gg$, PUL, 1991) et plusieurs articles, y compris dans cette revue, qui n'ont pas été étrangers aux développements engendrés dans le présent volume. La qualité de leurs réflexions passées et leur complémentarité féconde ont débouché sur un livre précis, d'une extrême clarté, aux références solides et multiples - juridiques d'abord, mais également extrajuridiques -, qui rend compte d'une réalité syndicale en mouvement et qui pose les jalons d'une meilleure intelligibilité d'une représentation organisée dans un environnement en transformation accélérée. 
Dès l'introduction, ils précisent le territoire d'investigation : le Québec, en Amérique du Nord. Le premier chapitre, «De la représentation syndicale » liée au salariat, décrit ses contradictions internes, le caractère polysémique de l'action syndicale, son évolution historique. Le deuxième chapitre porte sur « L'acteur syndical $\gg$ et ses tendances, au Québec en premier lieu, mais aussi au Canada et aux États-Unis. Le taux de syndicalisation au Québec et au Canada fait cruellement pâlir celui d'autres pays, les États-Unis, certes, qui a chuté fortement, quitte à montrer des signes de reprise ces derniers temps, mais, serais-je tenté d'ajouter, la France encore plus intensément, qui se situe en queue du peloton communautaire européen. Suit une étude fouillée du syndicalisme québécois, situé par rapport au Canada et aux États-Unis, avec le système d'accréditation et la tendance structurelle à la négociation fragmentée - qui, est-il dit plus loin, peut diminuer, dans certains cas, sous la pression du contexte économique - , la collaboration paritaire en matière d'emploi sur le plan pancanadien, la nécessité d'adaptation continue à l'égard des stratégies de gestion de la production et des ressources humaines, avec les réticences et les ouvertures qui s'ensuivent, la gamme des services offerts (et on ne le dira jamais assez pour un pays comme la France), l'action politique, enfin (qui détonne toujours avec les traductions qu'elle avait prises en Europe du Sud), pour aboutir aux incertitudes d'un accroissement prouvé de l'importance de la représentation syndicale et de son renforcement institutionnel. Comme quoi, il ne faut jamais se reposer sur ses lauriers, et toujours combattre, construire, innover...

Le chapitre 3 est consacré au régime général de représentation collective sur le plan de l'entreprise, et les auteurs reviennent avec plus de précisions sur l'unité d'accréditation et la négociation collective. La discussion des concepts d'entreprise et d'employeur uniques invite le lecteur à des comparaisons avec le débat existant dans des pays extra nord américains (en France particulièrement), comme celle, d'ailleurs, entreprise sur le précompte («évité » en France et en déclin en Italie) et sur les réunions sur les lieux de travail. La frontière, graduellement perméable, entre salarié et travailleur indépendant, la définition de l'«entrepreneur dépendant », celle du «salarié » et l'interprétation ductile de la subordination juridique, appellent, naturellement, pour le lecteur latino-européen, la référence au débat local autour du même sujet, y compris les échanges animés, particulièrement en Italie, autour des travailleurs « parasubordonnés ». Murray et Verge ne reviennent pas fortuitement sur la question dans le chapitre 5, «Futur visage juridique de la représentation syndicale », car, le futur étant déjà présent, elle constitue une des réalités les plus tangibles de notre actualité. La nature conflictuelle des relations du travail s'accompagne désormais d'une zone d'intérêts communs, qu'on peut rapprocher, sans déformer la pensée des écrivains, de la consensualisation des comportements économiques qu'ils évoquaient dans les premières pages du livre, exposée de façon plus circonstanciée dans le chapitre 5 .

Le chapitre 4 procède à une description complète de $\ll$ La représentation syndicale au-delà de l'entreprise » avec, notamment, le rôle de lobbisme des syndicats et un très utile tableau de la représentation du « salarié-citoyen » audelà de l'entreprise au Québec. Les quatre premiers chapitres préparent le saut dans l'avenir, annoncé dans le soustitre « Visage juridique actuel et futur ». Le chapitre 5, par conséquent, «Futur visage juridique de la représentation syndicale $»$, reprend, pour les développer, certaines incursions parsemées le long des parties précédentes, mais pose d'emblée une question essentielle : le changement en cours conduirait-il à la 
disparition de la représentation syndicale au Québec (et on pourrait dire autant de celles d'autres contrées) ? Le nouveau contexte est expliqué : la mondialisation, l'accord de libre-échange nordaméricain et celui, séparé, du Canada avec le Chili, la participation croissante des femmes au marché du travail, la croissance globale du taux de scolarisation, etc., modifient un paysage dans lequel les syndicats ont parfois du mal à s'intégrer complètement. Ainsi, les conseils qui leur sont prodigués (p. 111) méritent d'être retenus, dans les conditions, aussi, d'une transformation de la gestion de la production et de l'organisation du travail, de même que de l'externalisation de nombre d'activités des entreprises et de la main-d'œuvre correspondante.

Murray et Verge évoquent le réseau, qu'il s'agisse d'entreprise-réseau ou du réseau d'entreprises, avec ce que cela entraînerait comme représentation de réseau. Et on peut penser, pour ne prendre qu'un seul exemple, à des traductions juridiques souhaitées nées de l'évolution de l'impartition, adaptées à la création de parcs fournisseurs dans l'industrie automobile, ce qui nécessite que les co (ou sous) traitants, présents dans des installations de proximité, soient intégrés très en amont dans la conception d'un véhicule ou d'une usine et résultant, plus généralement, de l'addition des compétences, la mutation du partenariat, l'apparition du concept d'entreprise étendue et le partage (souhaité) des progrès technologiques et économiques au sein d'une culture de compétition très présente. Et que dire de la présence des personnels des fournisseurs sur les chaînes de montage du donneur d'ordre?

La représentation syndicale, avertissent les auteurs, ne se confine pas au salariat, l'action législative pouvant prendre en compte les intérêts de collectivités professionnelles qui, disent-ils, «bien que n'appartenant pas au monde traditionnel du travail salarié, en partagent de façon marquée une situation caractéristique de dépendance économique ». Les auteurs souhaitent, par ailleurs, une présence syndicale plus manifeste en amont des orientations stratégiques des entreprises, en préconisant, modérément, une participation aux organes juridiques des compagnies, et en ouvrant, ainsi, un débat sur un stakeholder particulier et la gouvernance d'entreprise.

L'exclusion de la notion de salarié de la strate de direction, qui renvoie dans nos pays européens du sud au débat similaire sur le cumul des situations de salarié et de dirigeant social, m'a remis en mémoire un jugement récent du «Jusgado de lo Social numéro 5 » de Madrid, qui a refusé à une ancienne dirigeante de la confédération syndicale des commissions ouvrières la qualité de salariée (el vinculo laboral). Un prétexte pour dire combien j'ai apprécié dans cet ouvrage l'ouverture comparatiste et disciplinaire.

Ouvrage parfaitement maîtrisé et parfaitement écrit (j'ai parlé plus haut d'écrivains), par deux pédagogues possédant non seulement les arcanes du droit du travail, mais une aisance équivalente dans les domaines connexes des sciences sociales. Ouvert sur l'avenir et accessible à un public plus étendu que d'autres productions académiques, il s'adresse autant aux lecteurs québécois, qu'à ceux qui, francophones, se retrouveront dans nombre de sujets abordés. Avec, en prime, une bibliographie, un index et un index de la législation.

DIMITRI WEISS

Institut d'administration des entreprises Université Paris I 